\title{
Relapsing Polychondritis Complicated by Giant Cell Myocarditis and Myositis
}

\author{
Mari Watanabe ${ }^{1}$, Hiroki Suzuki ${ }^{1}$, Tomoka Ara ${ }^{1}$, Midori Nishizuka ${ }^{1}$, Mami Morita ${ }^{1}$, \\ Chisa Sato ${ }^{1}$, Fumihiro Tsuchida ${ }^{1}$, Hiroaki Takeda ${ }^{1}$ and Tomoya Kato ${ }^{2}$
}

\begin{abstract}
An 83-year-old man presented with a three-week history of dyspnea. The clinical features suggested a diagnosis of relapsing polychondritis (RP); however, the patient died of heart failure. An autopsy revealed active chondritis of the tracheal and bronchial cartilage. Furthermore, giant cell myocarditis (GCM) and myositis were detected. To the best of our knowledge, this represents the first report of RP complicated by GCM and myositis. In patients with RP, GCM and myositis, CD163-positive macrophages and T-cells are most common, and the T-cell subset exhibits CD8 predominance. Common mechanisms of tissue damage caused by cytotoxic T-cells are likely to contribute to RP, GCM and myositis.
\end{abstract}

Key words: relapsing polychondritis, giant cell myocarditis, myositis, autoimmune disease, CD8+ lymphocytes

(Intern Med 52: 1397-1402, 2013)

(DOI: 10.2169/internalmedicine.52.9080)

\section{Introduction}

Relapsing polychondritis (RP) is a disease characterized by the recurrent and progressive inflammation of cartilage and tissue containing mucopolysaccharide and manifests with diverse clinical symptoms. Although the cause remains unknown, an autoimmune basis has been suggested. We encountered a case of RP complicated by giant cell myocarditis (GCM) and myositis. Complication by GCM and myositis is considered to be based on an autoimmune mechanism. We herein report our case and consider it to be important for understanding the pathogenic mechanisms of RP.

\section{Case Report}

An 83-year-old man presented with a three-week history of dyspnea. He had smoked 15 cigarettes per day for 40 years. He worked in a mine between the ages of 19 and 54 . He had been under treatment with oral medicines from a neighborhood hospital for bronchial asthma since 1999. The patient developed wheezing and coughing around mid-April 2009 and visited his neighborhood hospital where he re- ceived intravenous aminophylline and steroids. In early May, a repeat infusion of aminophylline/steroids failed to provide relief. The patient was referred to the emergency outpatient unit of our hospital for a detailed examination and treatment and was admitted.

The following results were obtained on a physical examination: a body temperature of $37.3^{\circ} \mathrm{C}$, an oxygen saturation $\left(\mathrm{SpO}_{2}\right)$ on room air of $95 \%$ and mild pallor of the palpebral conjunctiva. Inspection and palpation revealed no abnormal findings in the auricles or nose. There were no heart murmurs. Chest auscultation revealed both expiratory and inspiratory wheezing over the entire chest. Mild pitting edema was observed in both lower limbs.

The results of a blood examination were as follows: an $\mathrm{Hb}$ level of $11.8 \mathrm{~g} / \mathrm{dL}$, a white blood cell (WBC) count of 15,900 cells $/ \mu \mathrm{L}$, a serum C-reactive protein (CRP) level of $13.37 \mathrm{mg} / \mathrm{dL}$ and a serum sodium level of $129 \mathrm{mEq} / \mathrm{L}$. Tests for rheumatoid factor, antinuclear antibodies, antineutrophil cytoplasmic antibodies (ANCA) and anti-type II collagen antibodies were all negative. Electrocardiography revealed no abnormal findings. Respiratory function testing revealed a decreased ratio of the forced expiratory volume in 1 second/forced vital capacity (FEV1/FVC) of $57 \%$ and a peak

${ }^{1}$ Department of Respiratory Medicine, Saiseikai Yamagata Saisei Hospital, Japan and ${ }^{2}$ Department of Diagnostic Pathology, Yamagata University Faculty of Medicine, Japan

Received for publication October 8, 2012; Accepted for publication February 11, 2013

Correspondence to Dr. Mari Watanabe, mari_wata5055@yahoo.co.jp 


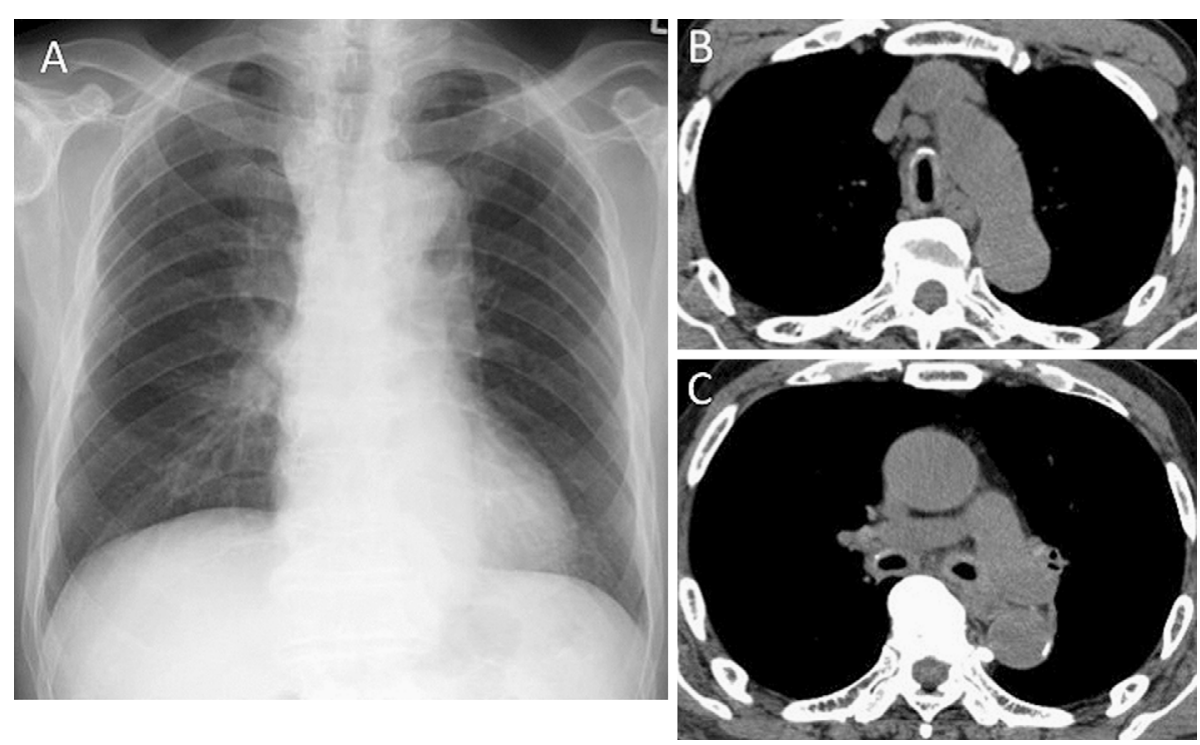

Figure 1. Chest radiography on admission showed tracheal stenosis (A). Chest computed tomography (CT) on admission showed thickening and narrowing of the trachea and also both main bronchi $(\mathrm{B}, \mathrm{C})$. Calcification of the tracheal cartilage was also noted.

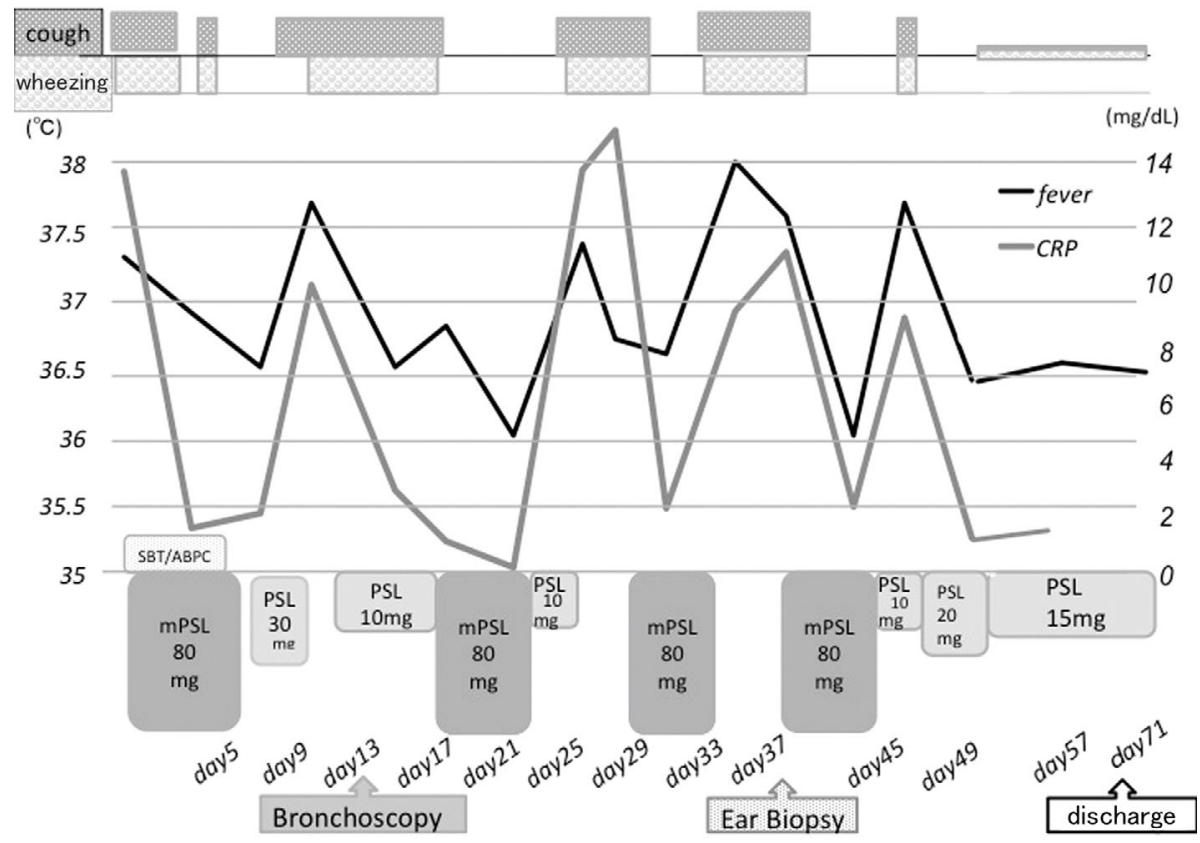

Figure 2. Clinical course.

expiratory flow rate of $43.5 \%$ of the predicted value.

A chest radiograph obtained on admission (Fig. 1A) revealed tracheal stenosis. Chest computed tomography (CT) performed on admission (Fig. 1B and C) revealed marked wall hypertrophy and stenosis of the trachea and bronchi, as well as partial calcification of the tracheal wall. 2-Fluoro-2deoxy-D-glucose positron-emission tomography (18F-FDG PET) performed during treatment with methylprednisolone (mPSL) did not reveal any apparent sites of FDG accumulation.

After admission, improvement of the patient's respiratory status and inflammatory findings was observed following treatment with mPSL and then prednisolone (PSL). How- ever, relapse occurred as soon as the dose was reduced (Fig. 2). A bronchoscopic examination (Fig. 3) revealed markedly reddened mucosa extending from the trachea to both main bronchi and blunting of the cartilaginous rings due to edema. In addition, the tracheal lumen was constricted in the form of a saber sheath. A biopsy of the bronchial mucosa distal to the main bronchi was performed, which revealed inflammatory cell infiltration, consisting primarily of lymphocytes, under the bronchial epithelium and around the bronchial glands. The administration of steroids was temporarily discontinued, and a biopsy of the ear cartilage was performed. However, no inflammatory cell infiltration was found around the cartilage. There was no evidence 


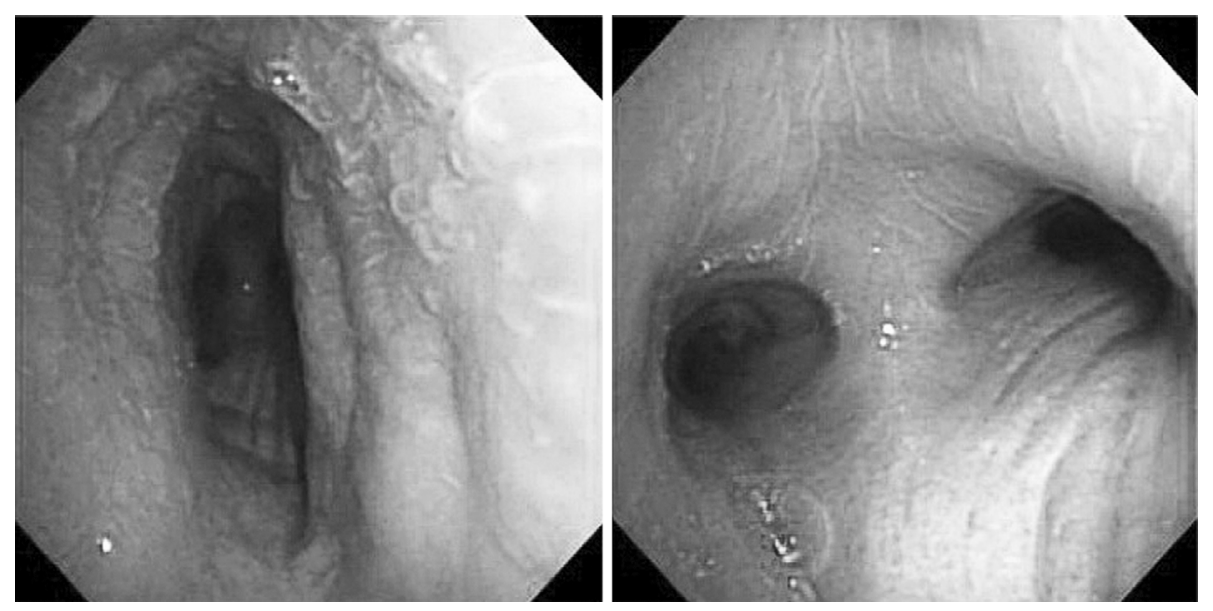

Figure 3. Bronchoscopic examination showed grossly edematous and reddish mucosa of the trachea and the main bronchi, blunting of the cartilaginous rings, and saber-sheath type tracheomalacia.

of either ocular inflammation or arthritis. Although the above findings did not satisfy the diagnostic criteria, RP was strongly suspected based on the CT findings, the bronchoscopic findings and the patient's clinical course. The dose of PSL was gradually tapered to $15 \mathrm{mg} / \mathrm{day}$, and the patient was discharged.

Four days after being discharged, the patient was brought back to the hospital by ambulance with consciousness disturbance. Upon arrival, the patient's condition was as follows: a body temperature of $39.0^{\circ} \mathrm{C}$, a blood pressure of $78 /$ $57 \mathrm{mmHg}$, a heart rate of $40-49 \mathrm{bpm}$ and an $\mathrm{SpO}_{2}$ of $90 \%$ (under $9 \mathrm{~L} /$ minute of oxygen via a reservoir mask with a bag). An electrocardiogram revealed $\mathrm{III}^{\circ}$ atrioventricular block; however, a plain chest radiograph revealed no marked changes from the previous image. The results of a blood test were as follows: a creatine kinase (CK) level of 2,790 IU/L, a CK-myoglobin (MB) level of $240 \mathrm{U} / \mathrm{L}$, an aspartate aminotransferase (AST) level of $550 \mathrm{IU} / \mathrm{L}$ and a lactate dehydrogenase (LDH) level of 1,665 IU/L, indicative of marked increases in the serum levels of myocardial enzymes. Moreover, the WBC count and serum CRP level were elevated to $10,600 / \mu \mathrm{L}$ and $10.6 \mathrm{mg} / \mathrm{dL}$, respectively. Echocardiography revealed hypokinesis of the anteroseptal wall, suggestive of an acute myocardial infarction. However, coronary angiography demonstrated no significant coronary artery stenosis. Although extracorporeal pacing was used to treat the $\mathrm{III}^{\circ}$ atrioventricular block, pacing failure occurred. The patient died eight hours after he was brought to the hospital. An autopsy was performed with the consent of his family.

\section{Autopsy findings}

The tracheal and bronchial mucosa exhibited redness and edematous swelling. A histopathological examination revealed band-like infiltration with neutrophils, lymphocytes and macrophages around the bronchial cartilage. Images of active chondritis with degeneration and destruction of the cartilage were observed (Fig. 4A). Similar findings were also observed in the costal cartilage. Sections of the heart revealed edematous myocardium. A histological examination revealed inflammatory cell infiltration, primarily consisting of lymphocytes and macrophages, among the cardiac myocytes. Evidence of myocarditis with degeneration and necrosis of the cardiac myocytes was observed over a wide area. Focal dislodgment of the cardiac myocytes was observed. Dense aggregation of inflammatory cells was observed. Multinucleated giant cells were also sparsely distributed. There was a small amount of fibril formation and lipid infiltration (Fig. 4B and C). Moreover, inflammatory cell infiltration, consisting primarily of lymphocytes and multinucleated giant cells, was observed in the tongue, esophagus, diaphragm and iliopsoas muscles (Fig. 4D). Along with acute myocarditis and cardiac failure, hepatic centrilobular necrosis suggestive of hemodynamic failure and systemic venous congestion of the lungs, liver, etc. were also observed.

Immunohistochemistry was performed on formalin-fixed, paraffin-embedded tissue. In RP, GCM and myositis, among the types of infiltrating inflammatory cells, CD163-positive macrophages and T-cells are most common, and the T-cell subset exhibits CD8 predominance (high power $\times 400$, represented by the mean value of five visual fields) (Figs. 5, 6). The primary antibodies used were as follows: CD3 (PS1 (Nichirei), prediluted, pan T-cell marker), CD4 (4B12 (Nichirei), prediluted), CD8 (C8/144B (Nichirei), prediluted), CD20 (L26 (DAKO), 1:200 dilution, pan B-cell marker), CD138 (MI15 (DAKO), 1:50 dilution, plasma cell marker), CD57 (HNK-1 (Becton-Dickinson), prediluted, NK cell marker), CD163 (10D6 (Novocastra), 1:400 dilution, macrophage marker), CD15 (C3D-1 (DAKO), 1:25 dilution, neutrophil marker), S-100 protein (polyclonal (Nichirei), prediluted, dendritic cell marker), DC-sign (polyclonal (Santa Cruz), 1:200 dilution, immature dendritic cell marker) and CD83 (1H4b (Novocastra), 1:50 dilution, mature dendritic cell marker). 


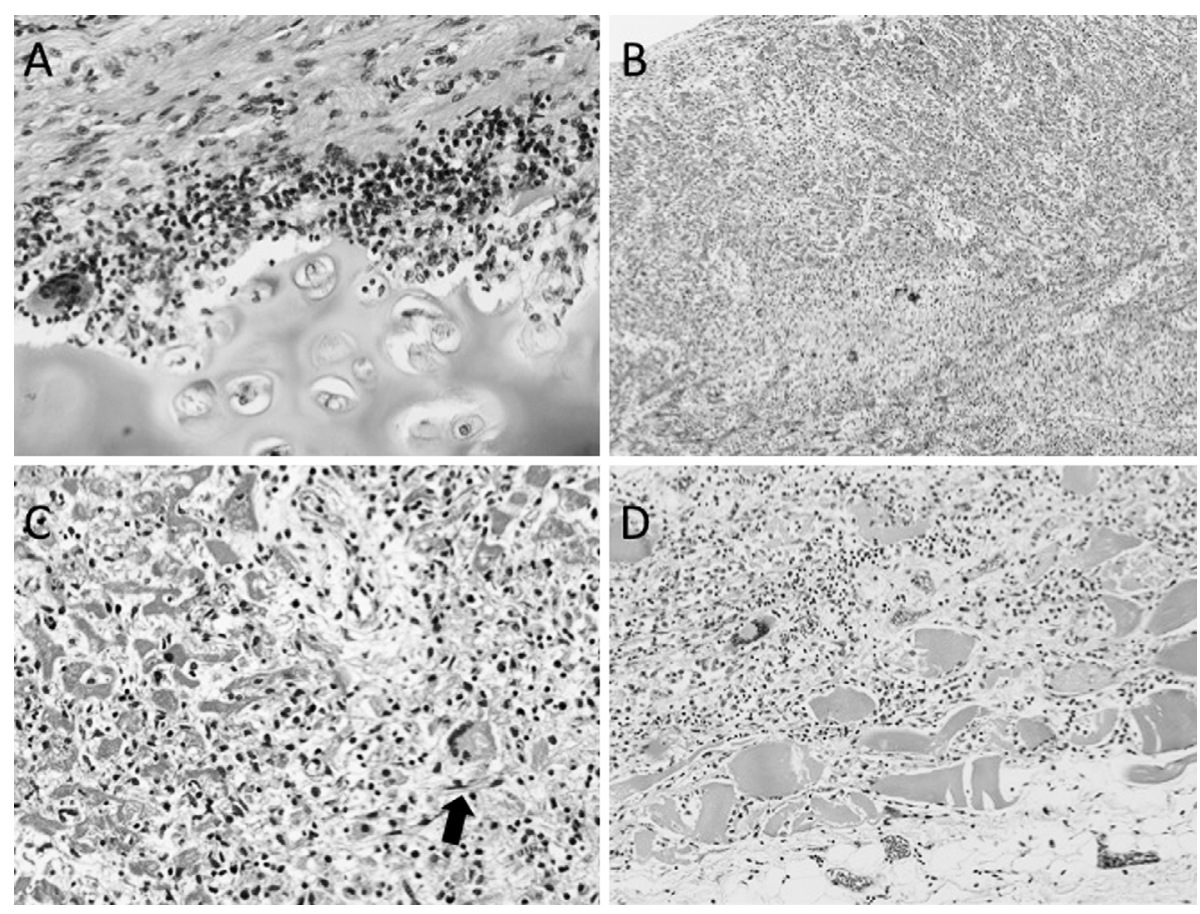

Figure 4. Histopathological findings of the tracheal specimens obtained at autopsy showed degeneration of the cartilaginous tissue accompanied by mononuclear cell and polymorphonuclear cell infiltration at the cartilage-soft tissue interface (A; Hematoxylin and Eosin staining, high-power field). Microscopic findings of the cardiac muscle showed marked inflammatory cell infiltration, consisting of lymphocytes, macrophages, and multinucleated giant cells (arrows), with myocardial degeneration and necrosis detected at autopsy (B; Hematoxylin and Eosin staining, low-power field. C; Hematoxylin and Eosin staining, high-power field). Microscopic findings of the diaphragm also showed inflammatory cell infiltration, mainly with lymphocytes. Multinucleated giant cells were also observed (D; Hematoxylin and Eosin staining, mid-power field).

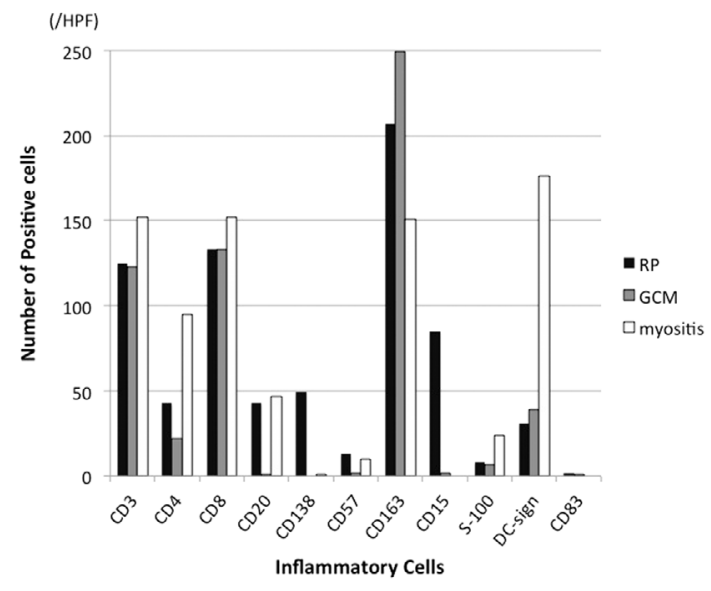

Figure 5. Comparison of inflammatory cells in RP, GCM, and myositis. In RP, GCM, and myositis, CD163-positive macrophages and $\mathrm{T}$ cells were most common, and the $\mathrm{T}$-cell subset showed CD8 predominance (high power $\times 400$, represented by the mean value in 5 visual fields).

\section{Discussion}

$\mathrm{RP}$ is characterized by recurrent and progressive inflammation of tissue containing cartilage and mucopolysaccha- ride and manifests with diverse clinical symptoms. This disease was first reported in 1923 as "polychondropathia" by Jaksch-Wartenhorst (1).

With respect to the diagnostic criteria of RP, McAdam proposed in 1976 that a definitive diagnosis could be made if three or more of the following criteria are fulfilled in addition to biopsy evidence: 1) bilateral auricular chondritis, 2) nonerosive inflammatory polyarthritis, 3) nasal chondritis, 4) ocular inflammation, 5) respiratory tract chondritis and 6) cochlear/vestibular dysfunction (2). Furthermore, Damiani indicated in 1979 that RP can be diagnosed if any of the following criteria are fulfilled: 1) three or more of the six criteria of McAdam, 2) one or more of the six criteria of McAdam and histological findings or 3) chondritis involving two or more anatomically separate sites that is responsive to treatment with steroids or dapsone (3). In our case, the autopsy revealed airway chondritis that was confirmed on histology. Because this fulfilled criterion 2) of Damiani, our patient was diagnosed as having RP.

In this case, GCM might have directly caused the death of the patient, based on the autopsy results. Meanwhile, RP complicated by myocarditis has been reported in only a review of three cases by Dolan et al. (4), and a search of the literature conducted to the best of our ability revealed no other reports of RP complicated by GCM. 


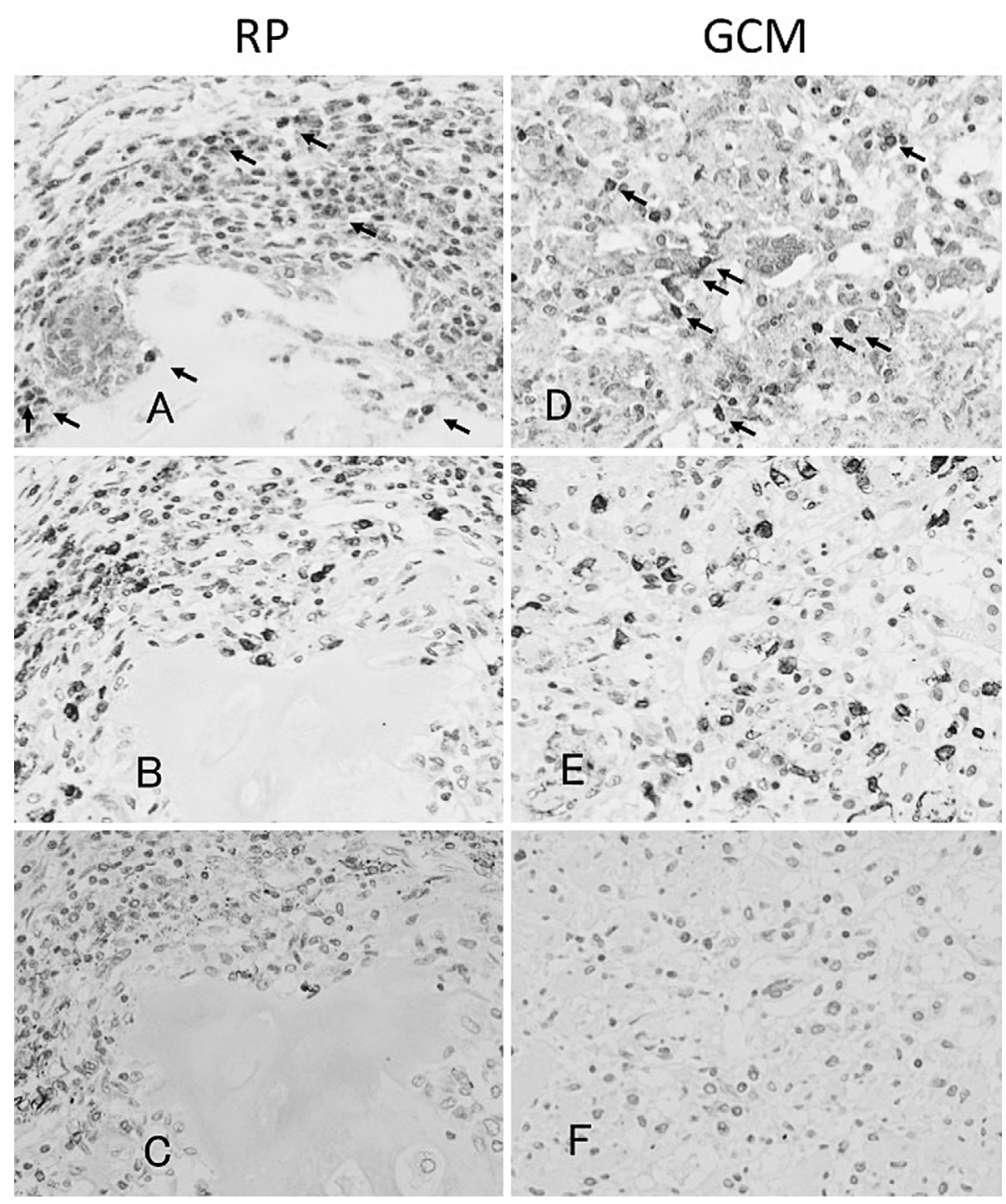

Figure 6. Immunohistochemical features of lymphocytes among infiltrating inflammatory cells in RP and GCM. In both RP and GCM, CD8+ lymphocytes (B and E) were abundant as compared with CD4+ lymphocytes (arrows) (A and D) and CD20+ lymphocytes (C and F). (Immunohistochemistry; high-power field).

GCM is a disease characterized by severe inflammation accompanied by the presence of multinucleated giant cells in cardiac myocytes. Although the incidence of this type of myocarditis is extremely low among cases of myocarditis, the reported frequency is in the range of $4 \%$ to $41 \%$, based on studies of severe myocarditis. The prognosis of giant cell myocarditis is extremely poor. Cooper et al. reported that $89 \%$ of their patients died or required cardiac transplantation and that the mean survival was 5.5 months (5). In cases of this disease complicated by ulcerative colitis, myasthenia gravis, aortitis syndrome, rheumatoid arthritis, etc., which occur with a rate of approximately $20 \%$, it is assumed that autoimmunity is involved as the pathogenic mechanism.

Moreover, myositis was also present in our case. In regard to RP complicated by myositis, there is only one previous report of RP complicated by dermatomyositis, reported by Arniaud et al. (6) Furthermore, the first report of coexistent GCM and myositis is that of thymoma complicated by myasthenia gravis and GCM/myositis reported by Giordano et al. (7) While five cases exhibiting this association have been reported in Japan, all of the patients presented with conduction disorders or myocardiopathy complicated by cardiac failure or sudden death within a short period. It is considered that production of autoantibodies against the myocardium/skeletal muscle is likely to be involved in this association. The involvement of ryanodine receptor antibodies, which are muscle calcium release receptor antibodies, has also been reported $(8,9)$. In addition, HooKim et al. demonstrated the presence of antibodies in the myocardium based on positive staining for anti-immunoglobulin G ( $\operatorname{IgG})$ myocardial antibodies on the surface of cardiac fibers (10).

At present, the causes of RP remain unknown. Meanwhile, cases positive for antinuclear antibodies or rheumatoid factor (11), cases positive for autoantibodies against anti-type II collagen antibodies, a primary component of cartilage, as well as anti-type IX and XI collagen antibod- 
ies (12-14) and cases positive for matririn-1 antibodies (15) have also been reported. Moreover, deposition of immunoglobulin and complement has been observed in cartilage (4). Furthermore, the frequency of detection of human leukocyte antigen-DR4 (HLA-DR4) has been reported to be high in these cases (16). Steroids and immunosuppressants are effective for treating this condition. There have also been reports of cases of RP complicated by many autoimmune diseases, such as vasculitic syndrome, rheumatoid arthritis, systemic lupus erythematosus and Sjögren's syndrome (2). Based on the above, the possibility of involvement of autoimmunity is strongly suspected.

We examined cell surface markers in the autopsied tissue of the present patient. While past reports of T-cell subsets have shown CD4 predominance in patients with RP (17) and CD8 predominance in patients with GCM (18), it is interesting that the RP observed in our patient exhibited CD8 predominance. In this case, the common mechanisms of tissue damage caused by cytotoxic T-cells are likely to have contributed to GCM, myositis and acute exacerbation of RP.

The pathogenesis of RP remains unknown. Accumulating cases with various associations and conducting detailed examinations of the autoimmune processes observed in such cases will be useful for elucidating the pathology of this disease.

The authors state that they have no Conflict of Interest (COI).

\section{References}

1. Jacksch-Wartenhorst R. Polychondropathia. Wien Arch Inn Med 6: 93-100, 1923.

2. McAdam LP, O'honlan ML, Bluestone R, et al. Relapsing polychondritis: prospective study of 23 patients and a review of the literature. Medicine 55: 193-216, 1976.

3. Damiani JM, Levine HL. Relapsing polychondritis. Laryngoscope 89: 929-946, 1979.

4. Dolan DL, Lemmon GB, Teitelbaum SL. Relapsing polychondritis analytical review and studies on pathogenesis. Am J Med 41: 285299, 1966.
5. Cooper LT Jr, Berry Gj, Shabetai R. Idiopathic giant-cell myocarditis -natural history and treatment. N Engl J Med 336: 18601866, 1997.

6. Arniaud D, Mattei JP, Pham T, et al. Coexistent dermatomyositis, relapsing polychondritis, and positive lyme serology. Revue du rhumatisme [Engl.Ed.] 64: 589-596, 1997.

7. Giordano A, Haymond J. Myasthenia gravis report cases with necropsy findings. Am J Clin Pathol 14: 253, 1944.

8. Mygland A, Vincent A, Newsom-Davis J, et al. Autoantibodies in thymoma-associated myasthenia gravis with myositis or neuromyotonia. Arch Neurol 57: 527-531, 2000.

9. Suzuki S, Utsugisawa K, Yoshikawa H, et al. Autoimmune targets of heart and skeletal muscles in myasthenia gravis. Arch Neurol 66: 1334-1338, 2009.

10. HooKim K, deRoux S, Igbokwe A, et al. IgG anti-cardiomyocyte antibodies in giant cell myocarditis. Ann Clin Lab Sci 38: 83-87, 2008.

11. Watanabe $\mathrm{Y}$, Miwa $\mathrm{C}$, Tubochi $\mathrm{H}$, et al. A case of airway-limiting type relapsing polychondritis. Nihon Kokyuki Gakkai Zasshi 45: 987-991, 2007 (in Japanese, Abstract in English).

12. Foidart JM, Abe S, Martin GR, et al. Antibodies to type II collagen in relapsing polychondritis. N Eng J Med 229: 1203-1207, 1978.

13. Terato K, Shimozuru Y, Katayama K, et al. Specificities to antibodies to type II collagen in rheumatoid arthritis. Arthritis Rheum 33: 1493-1500, 1990.

14. Alsalameh S, Mollenhauer J, Scheuplein F, et al. Preferential cellular and humoral immune reactivities to native and denatured collagen type IX and XI in a patient with fatal relapsing polychondritis. J Rheumatol 20: 1419-1424, 1993.

15. Hansson AS, Heinegard D, Piette JC, et al. The occurrence of autoantibodies to matrilin 1 reflects a tissue-specific response to cartilage of the respiratory tract in patients with relapsing polychondritis. Arthritis Rheum 44: 2402-2412, 2001.

16. Lang B, Rothenfusser A, Lanchbury JS, et al. Susceptibility to relapsing polychondritis is associated with HLA-DR4. Arthritis Rheum 36: 660-664, 1993.

17. Ouchi N, Uzuki M, Kamataki A, Miura Y, Sawai T. Cartilage destruction is partly induced by the internal proteolytic enzymes and apoptotic phenomenon of chondrocytes in relapsing polychondritis. J Rheumatol 38: 730-737, 2011.

18. Litovsky SH, Burke AP, Virmani R. Giant cell myocarditis: an entity distinct from sarcoidosis characterized by multiphasic myocyte destruction by cytotoxic $\mathrm{T}$ cells and histiocytic giant cells. Mod Pathol 9: 1126-1134, 1996.
(C) 2013 The Japanese Society of Internal Medicine http://www.naika.or.jp/imonline/index.html 